\title{
THE FLOW ANALYSIS OF FIUIDS IN FRACTAL RESERVOIR WITH THE FRACTIONAL DERIVATIVE*
}

\author{
TIAN Ji \\ Institute of Mechanics, Chinese Academy of Science,Beijing 100080,China \\ Gudong Petroleum Factory of Shengli Petroleum Administration, Dongying 256504,China \\ TONG Deng-ke \\ Department of Applied Mathematics, China University of Petroleum, Dongying 257061, China, \\ E-mail:tongdk@mail.hdpu.edu.cn
}

(Received Apr. 2, 2004)

\begin{abstract}
In this paper, fractional order derivative, fractal dimension and spectral dimension are introduced into the seepage flow mechanics to establish the flow models of fluids in fractal reservoirs with the fractional derivative. The flow characteristics of fluids through a fractal reservoir with the fractional order derivative are studied by using the finite integral transform, the discrete Laplace transform of sequential fractional derivatives and the generalized Mittag-Leffler function. Exact solutions are obtained for arbitrary fractional order derivative. The long-time and short-time asymptotic solutions for an infinite formation are also obtained. The pressure transient behavior of fluids flow through an infinite fractal reservoir is studied by using the Stehfest's inversion method of the numerical Laplace transform. It shows that the order of the fractional derivative affect the whole pressure behavior, particularly, the effect of pressure behavior of the early-time stage is larger The new type flow model of fluid in fractal reservoir with fractional derivative is provided a new mathematical model for studying the seepage mechanics of fluid in fractal porous media.
\end{abstract}

KEYWORDS: fractional calculus, porous media, fractal, integral transform exact solution .

\section{INTRODUCTION}

Chang and Yortsos ${ }^{[1]}$ presented the theoretical model for infinite fractal reservoir. Acuna et al. ${ }^{[2]}$ explained the fractal characteristic of a naturally fractured geothermal field. Acuna ${ }^{[3]}$ reviewed the theoretical background of fractal analysis. They also demonstrated the application of various diagnostic techniques for fractal pressure transient analysis as developed by Chang and Yortsos ${ }^{[1]}$.Tong ${ }^{[4-7]}$ give the analytical solution of various cases for transient flow of fluid through a cylinder-source well of fractal reservoir.

In the flow of fluid in fractal reservoir, the dimensionless flow equation is written as ${ }^{[1]}$

$$
\frac{\partial p_{D}}{\partial t_{D}}=\frac{1}{r_{D}^{d_{f}-1}} \frac{\partial}{\partial r_{D}}\left(r_{D}{ }^{\beta} \frac{\partial p_{D}}{\partial r_{D}}\right)
$$

For the most porous media, the above equation can basically reflect the flow characteristics of fluids in fractal reserveoir. Acuna ${ }^{[2,3]}$ studied the flow characteristic of fluids in fractal reservoir from theory and production practice by using the above Eq. (1). In recent years, fractional calculus is as dynamic basis of the fractal geometry and fractional dimension. Fractional calculus has encountered much success in describing the constitutive relationship of viscoelastic fluid. The starting point of fractional order derivative model of non-Newtonian fluid is usually a classical differential equation that is modified by replacing the time derivative of an integer order by the so-called Riemann-Liouville fractional calculus operators.

*Project supported by the China National 973 Program (Grant No: 2002CB211708) and the Natural Science Foundation of Shandong Province (Grant No: Y2003F01).

Boigraphy: TIAN Ji (1964-), Male, Senior Engineering 
Thus the description of the fractional order constitutive relationship of viscoelastic fluid is more extensive. Friedrich ${ }^{[8]}$, Huang ${ }^{[9]}$, Tan ${ }^{[10]}$, $\mathrm{Xu}^{[11,12]}$ and Tong ${ }^{[13]}$ introduced fractional calculus in the rheology and analyzed various problems. They think that the fractional calculus approach is more appropriate for the viscoelastic fluid. Park ${ }^{[14]}$ introduced fractional calculus in the flow equation of fluid in fractal reservoir. He deem the fractional calculus approach tally with real fractal reservoir, particularly, the described pressure behavior of the early-time stage is more accurate. Jiang ${ }^{[15]}$ applied fractional calculus to the experimental data of the viscoelastic glue fluid to obtain a very good fit. Generally, fractional derivative is introduced in the flow model of fluid in fractal reserveoir, the flow equation is written as

$\frac{\partial^{\alpha} p_{D}}{\partial t_{D}^{\alpha}}=\frac{1}{r_{D}{ }^{{ }_{f}-1}} \frac{\partial}{\partial r_{D}}\left(r_{D}{ }^{\beta} \frac{\partial p_{D}}{\partial r_{D}}\right)$

where $D^{\alpha}=\frac{\partial^{\alpha}}{\partial t^{\alpha}}$ is fractional calculus operator of order $\alpha$ with respect to $t$ respectively and is defined as

$$
D^{\alpha}[y(t)]=\frac{1}{\Gamma(1-\alpha)} \frac{\mathrm{d}}{\mathrm{d} t} \int_{0}^{t} \frac{y(z)}{(t-z)^{\alpha}} \mathrm{d} z,
$$

$$
0 \leq \alpha \leq 1
$$

where $\Gamma(x)$ is Gamma function. While $\alpha=1$, it may be simplified as Eq. (1).

The aim of this paper is to study the flow of fluid in fractal reservoir with the fractional derivative. While $\alpha$ is arbitrary fraction, the exact solution for the flow equation is obtained by using the Laplace transform of fractional order derivative and the generalized Mittag-Leffler function. Many previous and classical results can be considered as special cases of our results. For example when $\alpha=1, d_{f}=2$, $\theta=0$ our results become Tong's solution ${ }^{[4]}$ for the flow of fluid in fractal reservoir. The flow characteristic and pressure sensitive to parameters of the fluids through fractal reservoir with fractional derivative are discussed by using the numerical inversion of Laplace transform and asymptotic solutions. It shows that the pressure characteristics of the fluid is sensitive to the order of the fractional derivative.

\section{THE FLOW MODEL OF FLUID IN FRACTAL RESERVOIR FRACTIONAL DERIVATIVE}

We assume the fractal permeable network embedded in impermeable Euclidean matrix, where the fractal network dimension is $d_{f}$, and the Euclidean matrix dimension is $d(d=1,2,3)$. Fluid flow only occurs in a fractal network. The material balance equation can be derived using integral relation ${ }^{[14]}$

$$
\begin{aligned}
& -\int_{0}^{t_{D}} Q_{r_{D}}\left(r_{D}, \tau_{D}\right) \mathrm{d} \tau_{D}= \\
& \quad \int_{0}^{t_{D}} K\left(t_{D}, \tau_{D}\right) \mathrm{d} \tau_{D} \int_{0}^{r_{D}} p_{D}\left(r_{D}^{\prime}, \tau_{D}\right) \mathrm{d} r_{D}^{\prime}
\end{aligned}
$$

The dimensionless total radial flow is

$$
Q_{r_{D}}=-r_{D}^{d_{f}-\theta-1} \frac{\partial}{\partial r_{D}} p_{D}\left(r_{D}, t_{D}\right)
$$

Equation (3) expresses the total radial flow passed up to time $t_{D}$ at distance $r_{D}$ from the well as a convolution integral of total pressure with the diffusion kernel. $d_{f}$ is fractal dimension and $\theta$ is diffusion exponent. Assuming the stationary process,

$$
K\left(t_{D}, \tau_{D}\right)=K\left(t_{D}-\tau_{D}\right)
$$

We applied the theory of fractional derivative to incorporate the memory. Combination of Eqs.(3) and (4) gives

$$
\begin{gathered}
\frac{\partial}{\partial t_{D}} \int_{0}^{t_{D}} K\left(t_{D}-\tau_{D}\right) p_{D}\left(r_{D}, \tau_{D}\right)= \\
\frac{1}{r_{D}^{d_{f}-1}} \frac{\partial}{\partial r_{D}}\left(r_{D}^{d_{f}-\theta-1} \frac{\partial p_{D}}{\partial r_{D}}\right)
\end{gathered}
$$

Eq.(5) can be interpreted as the second type of fractional diffusion equation ${ }^{[14]}$.

Therefore,Eq.(5) can be expressed as the following integro-differential equation

$$
\frac{\partial^{\alpha} p_{D}}{\partial t_{D}^{\alpha}}=\frac{1}{r_{D}^{d_{f}-1}} \frac{\partial}{\partial r_{D}}\left(r_{D}^{\beta} \frac{\partial p_{D}}{\partial r_{D}}\right)
$$

where 


$$
\begin{aligned}
& p_{D}=\frac{a V_{s} m\left(p_{0}-p\right)}{Q \mu r_{w}{ }^{1-\beta}}, t_{D}=\frac{m t}{\mu c_{f} r_{w}^{\theta+2}}, \\
& r_{D}=\frac{r}{r_{w}}, \beta=d_{f}-\theta-1
\end{aligned}
$$

with the initial condition

$$
\left.p_{D}\right|_{t_{D}=0}=0
$$

the inner boundary condition

$$
\left.\frac{\partial p_{D}}{\partial r_{D}}\right|_{r_{D}=1}=-1
$$

and the outer boundary condition

$$
\lim _{r_{D} \rightarrow \infty} p_{D}=0
$$

or

$$
\left.\frac{\partial p_{D}}{\partial r_{D}}\right|_{r_{D}=R}=0
$$

or

$$
\left.p_{D}\right|_{r_{D}=R}=0
$$

\section{THE EXACT SOLUTION FOR THE FLOW MODEL OF FLUID IN FRACTAL RESERVOIR \\ WITH FRACTIONAL DERIVATIVE}

3.1 Exact solution for the flow model of fluid in an infinite fractal reservoir

The initial and boundary value problem $I$ that is made up of Eqs. (6), (7),(8) and (9) represents the case of constant production rate from an infinite reservoir. We consider the initial value condition of integer order for fractional differential equation. The operator $D^{\alpha}$ is taken as the Miller-Ross sequential fractional derivative.Let

$$
\bar{p}_{D}\left(r_{D}, s\right)=\int_{0}^{\infty} e^{-s t_{D}} p_{D}\left(r_{D}, t_{D}\right) \mathrm{d} t_{D}
$$

Application of the Laplace transformation to Eqs.(6),(7),(8) and (9) yields

$$
s^{\alpha} \bar{p}_{D}=\frac{1}{r_{D}^{d_{f}-1}} \frac{\partial}{\partial r_{D}}\left(r_{D}^{\beta} \frac{\partial \bar{p}_{D}}{\partial r_{D}}\right)
$$

$$
\left.\frac{\partial \bar{p}_{D}}{\partial r_{D}}\right|_{r_{D}=1}=-\frac{1}{S}
$$

$$
\lim _{r_{D} \rightarrow \infty} \bar{p}_{D}=0
$$

Assume

$$
\bar{p}_{D}=r_{D}^{\frac{1-\beta}{2}} f(\rho), \rho=b r_{D}^{a}
$$

It can be shown that

$$
s^{\alpha} f=\frac{\partial^{2} f}{\partial \rho^{2}}+\frac{1}{\rho} \frac{\partial f}{\partial \rho}-\frac{v^{2}}{\rho^{2}} f
$$

where $\quad v=1-d_{s} / 2\left(d_{s}=\frac{2 d_{f}}{\theta+2}\right)$

$$
\left.\left[\frac{\partial f}{\partial \rho}+\frac{(1-\beta)}{2} f\right]\right|_{\rho=b}=-\frac{1}{s}
$$

$$
\bar{p}_{D}=\frac{r_{D}^{\frac{1-\beta}{2}} K_{v}\left(b s^{\frac{\alpha}{2}} r_{D}^{a}\right)}{s^{\frac{\alpha}{2}+1} K_{v-1}\left(b s^{\frac{\alpha}{2}}\right)}
$$

Eq.(18) is reduced to the following form at the wellbore $\left(r_{D}=1\right)$

$$
\bar{p}_{w D}=\frac{K_{v}\left(b s^{\frac{\alpha}{2}}\right)}{s^{\frac{\alpha}{2}+1} K_{v-1}\left(b s^{\frac{\alpha}{2}}\right)}
$$

The Weber transformation is given by ${ }^{[16]}$

$$
\bar{f}=W[f]=\int_{b}^{\infty} \rho f(\rho) \varphi_{1}(\rho, \lambda) \mathrm{d} \rho
$$

where

$$
\varphi_{1}(\rho, \lambda)=J_{v}(\lambda \rho) \quad Y_{v-1}(b \lambda)-Y_{v}(\lambda \rho) J_{v-1}(b \lambda)
$$

Applying the generalized Weber transformation to Eqs.(16), (17) yields 


$$
\bar{f}=\frac{2}{\pi \lambda s\left(\lambda^{2}+s^{\alpha}\right)}
$$

The inverse of Weber transformation can be expressed as ${ }^{[16]}$

$$
f(\rho)=\int_{0}^{\infty} \frac{\lambda \bar{f} \varphi_{1}(\rho, \lambda) \mathrm{d} \lambda}{J_{v-1}^{2}(b \lambda)+Y_{v-1}^{2}(b \lambda)}
$$

Substituting (21) into (15) the following expression can be obtained

$$
\begin{aligned}
& \bar{p}_{D}\left(r_{D}, s\right)= \\
& \int_{0}^{\infty} \frac{2 r_{D}^{\frac{1-\beta}{2}} \varphi_{1}\left(b r_{D}^{a}, \lambda\right) \mathrm{d} \lambda}{\left[J_{v-1}^{2}(b \lambda)+Y_{v-1}^{2}(b \lambda)\right] \pi s\left(\lambda^{2}+s^{\alpha}\right)}= \\
& \frac{2 r_{D}^{\frac{1-\beta}{2}}}{\pi} \int_{0}^{\infty} \frac{\bar{A}(\lambda, s) \varphi_{1}\left(b r_{D}^{a}, \lambda\right) \mathrm{d} \lambda}{\left[J_{v-1}^{2}(b \lambda)+Y_{v-1}^{2}(b \lambda)\right]}
\end{aligned}
$$

where $\bar{A}(\lambda, s)=\frac{1}{s\left(s^{\alpha}+\lambda^{2}\right)}$

Analytical solution for the initial and boundary value problem can be obtained as long as the inverse of Laplace transform of the function $\overline{\mathrm{A}}(\lambda, \mathrm{s})$. We will apply the discrete inverse Laplace transform method to give the analytical solution. Firstly, we used the following property of M-L function ${ }^{[17]}$

$$
L^{-1}\left[\frac{1}{s^{\alpha}+\lambda^{2}}\right]=t_{D}^{\alpha-1} E_{\alpha, \alpha}\left(-\lambda^{2} t_{D}^{\alpha}\right)
$$

in which $\mathrm{E}_{\mu, v}(\mathrm{z})$ denotes Mittag-Leffler function. Using Laplace transform the integral of convolution, we get

$$
\begin{gathered}
L^{-1}\left[\frac{1}{s\left(s^{\alpha}+\lambda^{2}\right)}\right]=\int_{0}^{t_{D}} \tau^{\alpha-1} E_{\alpha, \alpha}\left(-\lambda^{2} \tau^{\alpha}\right) \mathrm{d} \tau= \\
t_{D}^{\alpha} E_{\alpha, \alpha+1}\left(-\lambda^{2} t_{D}^{\alpha}\right)
\end{gathered}
$$

The exact solution for the flow model of an infinite formation is obtained by using Eq. (24)

$$
\begin{aligned}
& p_{D}\left(r_{D}, t_{D}\right)=\frac{2 r_{D}^{\frac{1-\beta}{2}} t_{D}^{\alpha}}{\pi} \bullet \\
& \int_{0}^{\infty} \frac{\varphi_{1}\left(r_{D}^{a}, \lambda\right) E_{\alpha, \alpha+1}\left(-\lambda^{2} t_{D}^{\alpha}\right) \mathrm{d} \lambda}{\left[J_{v-1}^{2}(b \lambda)+Y_{v-1}^{2}(b \lambda)\right]}
\end{aligned}
$$

If one sets $\alpha=1$ formula (25) can be reduced to the exact solution for the flow of fluid in an infinite fractal reservoir ${ }^{[4]}$

$$
\begin{gathered}
p_{D}=\frac{2 r_{D}^{\frac{1-\beta}{2}}}{\pi} \int_{0}^{\infty} \frac{\left(1-e^{-\lambda^{2} t_{D}}\right) M(\lambda) \mathrm{d} \lambda}{\lambda^{2}\left[J_{v-1}^{2}(b \lambda)+Y_{v-1}^{2}(b \lambda)\right]} \\
M(\lambda)=J_{v}\left(b r_{D}^{a} \lambda\right) Y_{v-1}(b \lambda)- \\
J_{v-1}(b \lambda) Y_{v}\left(b r_{D}^{a} \lambda\right)
\end{gathered}
$$

\subsection{Asymptotic solution}

(1) By using the property of Laplace transform, the following equality holds true for the final state $(s \rightarrow 0)$

$$
\begin{gathered}
K_{v}\left(b s^{\frac{\alpha}{2}}\right)=\frac{2^{-\delta} \Gamma(v)}{\left(b s^{\frac{\alpha}{2}}\right)^{v}}, \\
K_{v-1}\left(b s^{\frac{\alpha}{2}}\right)=K_{1-v}\left(b s^{\frac{\alpha}{2}}\right)= \\
K_{\delta}\left(b s^{\frac{\alpha}{2}}\right)=\frac{2^{\delta-1} \Gamma(\delta)}{\left(b s^{\frac{\alpha}{2}}\right)^{\delta}}
\end{gathered}
$$

where $\delta=\frac{d_{f}}{\theta+2}$. Substituting the above equations into equation (19), the long-time approximate solution in real space can be obtained by using the approximate formula of the modified Bessel function.

$$
\begin{gathered}
p_{w D}\left(t_{D}\right)=\frac{(\theta+2)^{2 v-1} \Gamma(v)}{\Gamma(1+\alpha v) \Gamma\left(\frac{d_{s}}{2}\right)} t_{D}^{\alpha v}, v \neq 0 \\
p_{w D}\left(t_{D}\right)=\frac{1}{\theta+2}\left[\alpha \ln t_{D}+2 \ln (\theta+2)-c\right], \\
v=0
\end{gathered}
$$


where $C$ is Euler's constant.

Eq.(27)indicates that the long-time pressure drop of crude oil can be used in computing fractal and formation parameters

$$
\begin{aligned}
& p_{0}-p(t)=\Delta p=C_{1} t^{\alpha v} \\
& C_{1}=\frac{Q(\theta+2)^{2 v-1} \Gamma(v) \mu^{1-v}}{a V_{s} C_{f}^{v} m^{1-v} \Gamma(1-v) \Gamma(1+\alpha v)}
\end{aligned}
$$

Eq. (29) exhibits that a plot of the pressure transient in logarithmic coordinates yields a straight line with the slope $\alpha V$ and the interception. $\log C_{1}$. The analysis of the drawdown test gets $v$.

(2) At the early-time $(s \rightarrow \infty)$, the following equality is tenable

$$
\bar{p}_{w D}=\frac{1}{s^{\frac{\alpha}{2}+1}}
$$

The solution in real space can be given by

$$
p_{w D}=\frac{t_{D}^{\frac{\alpha}{2}}}{\Gamma\left(\frac{\alpha}{2}+1\right)}
$$

The short-time asymptotic solution shows that a plot of the pressure transient in logarithmic coordinates yields straight lines with slope $\alpha / 2$. The physical meaning of the Eq.(30) is that well test data of oil field reveal the effect of fractional derivative in a short time. Then the order $\alpha$ of fractional derivative can be determined.

The pressure distributions are obtained by applying the Stehfest's method of the numerical inversion of Laplace transform to Eq. (19). The pressure behavior is described as:

(1) The dimensionless pressure solution exhibits two line segments in the lg-lg plot of the $P_{w D} \sim t_{D}$ (see Figs.1,2)

(a) The straight line with the slope $\alpha / 2$ reflects the effect of the fractional derivative.

(b) The straight line with the slope $\alpha v=\alpha\left(1-d_{s} / 2\right)$ reflects the effect of spectral dimension and the interception reveals the effect of the formation parameters.

(2) The pressure curves are influenced by the $\alpha$. The effects of $\alpha$ is on the whole flow stages. In the initial stage, the larger the value of $\alpha$, the smaller dimensionless pressure is. With time, the pressure curves show sudden variations and pressure curves intersect at a point. The dimensionless pressure henceforth is smaller for a smaller value of $\alpha$. This is the reason why the pressure at initial stages is larger for the smaller order of corresponding fractional derivative. The pressure change is slower in the whole flow process. The pressure at initial stages is smaller for the larger order of corresponding fractional derivative. With time, the pressure rises so fast that it will exceed the pressure of the smaller order of corresponding fractional derivative, thus pressure curves appear to change suddenly in the Fig.1.The pressure curves diverge from each other in later stages.

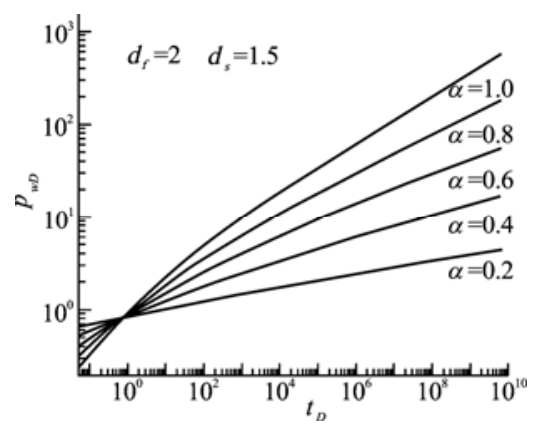

Fig.1 Effect of $\alpha$ on plot of pressure curve

(3) The effects of spectral dimension $d_{s}$ are mainly in later stages. The dimensionless pressure at any time is smaller for a greater value of $d_{s}$ (Fig.2).

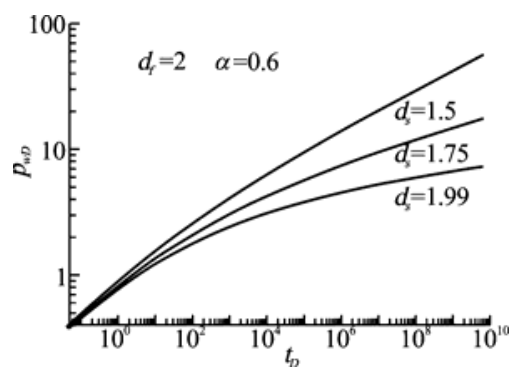

Fig.2 Effect of $d_{s}$ on plot of pressure curve

3.3 Exact solution for the flow model of fluid in a finite closed reservoir

The initial and boundary value problem II that is made up of Eqs. (6),(7),(8) and (10) represents the case of constant production rate from a finite closed reservoir. With the application of Laplace transformation to the initial and boundary value II , one obtains the boundary value problem that is made 
up of the Eqs. (12) (13) and (31)

$$
\left.\frac{\partial \bar{p}_{D}}{\partial r_{D}}\right|_{r_{D}=R}=0
$$

In order to gain the analytical solution of model II , we apply the following finite integral transform to the Eq.(12)

$\tilde{\bar{p}}_{D}(\lambda, s)=\int_{1}^{R} r_{D}^{d_{f}-1} \bar{p}_{D}\left(r_{D}, s\right) \varphi_{2}\left(\lambda_{n}, r_{D}\right) d r_{D}$

The inversion transform of $\widetilde{\bar{p}}_{D}\left(\lambda_{n}, s\right)$ may be written as ${ }^{[18]}$

$\bar{p}_{D}\left(r_{D}, s\right)=\sum_{n=0}^{\infty} \tilde{\bar{p}}_{D}\left(\lambda_{n}, s\right) \frac{\varphi_{2}\left(\lambda_{n}, r_{D}\right)}{N_{2}\left(\lambda_{n}\right)}$

where

$$
\begin{gathered}
\varphi_{2}\left(\lambda_{n}, r_{D}\right)=\left\{\begin{array}{l}
r_{D}^{\frac{1-\beta}{2}} R^{a-1} M_{1}\left(\lambda_{n} r_{D}\right),(n=1,2, \cdots) \\
1, n=0
\end{array}\right. \\
M_{1}\left(\lambda_{n} r_{D}\right)=\left[Y_{v-1}\left(b \lambda_{n}\right) J_{v}\left(b \lambda_{n} r_{D}^{a}\right)-\right. \\
\left.J_{v-1}\left(b \lambda_{n}\right) Y_{v}\left(b \lambda_{n} r_{D}^{a}\right)\right] \lambda_{0}=0, \lambda_{n}(n=1,2, \ldots)
\end{gathered}
$$

is the $n$-th positive root of the following equation

$$
J_{v-1}(b \lambda) Y_{v-1}\left(b \lambda R^{a}\right)-Y_{v-1}(b \lambda) \bullet
$$

$$
\begin{gathered}
J_{v-1}\left(b \lambda R^{a}\right)=0 \\
\frac{1}{N_{2}\left(\lambda_{n}\right)}=\left\{\begin{array}{l}
\frac{\pi^{2} b}{2} N\left(\lambda_{n}\right), n=1,2, \cdots \\
\frac{d_{f}}{R^{d_{f}}-1}, n=0
\end{array}\right. \\
N\left(\lambda_{n}\right)=\frac{\lambda_{n}^{2} J_{v-1}^{2}\left(b \lambda_{n}\right)}{R^{2 a-2}\left[J_{v-1}^{2}\left(b \lambda_{n}\right)-J_{v-1}^{2}\left(b \lambda_{n} R^{a}\right)\right]}
\end{gathered}
$$

The application of integral transform (32) to Eq. (12), by using boundary conditions (11),(31), we have

$$
\tilde{\bar{p}}_{D}\left(\lambda_{n}, s\right)=-\frac{2 R^{a-1} J_{v-1}\left(b \lambda_{n} R^{a}\right)}{\pi s b \lambda_{n} J_{v-1}\left(b \lambda_{n}\right)\left(\lambda_{n}^{2}+s^{\alpha}\right)}
$$
get

Substituting Eqs. (34) and (35) into Eq. (33), one

$$
\bar{p}_{D}\left(r_{D}, s\right)=
$$

$$
\frac{d_{f}}{R^{d_{f}}-1} \bar{p}_{0}\left(r_{D}, s\right)-\sum_{n=1}^{\infty} \bar{A}_{1}\left(\lambda_{n}, s\right) \bullet
$$

$$
\frac{\pi \lambda_{n} J_{v-1}\left(b \lambda_{n} R^{a}\right) J_{v-1}\left(b \lambda_{n}\right) \varphi_{2}\left(\lambda_{n}, r_{D}\right)}{J_{v-1}^{2}\left(b \lambda_{n}\right)-J_{v-1}^{2}\left(b \lambda_{n} R^{a}\right)}
$$

where

$$
\begin{aligned}
& \bar{p}_{0}\left(r_{D}, s\right)=\frac{1}{s^{\alpha+1}}, \bar{A}_{1}\left(\lambda_{n}, s\right)=\frac{1}{s^{1+\alpha}+\lambda_{n}^{2} s} \\
& p_{0}\left(r_{D}, t_{D}\right)=L^{-1}\left(\frac{1}{s^{\alpha+1}}\right)=\frac{t_{D}^{\alpha}}{\Gamma(\alpha+1)}
\end{aligned}
$$

The exact solution of the model II is obtained by using Eqs. (36) and (24)

$$
p_{D}\left(r_{D}, t_{D}\right)=\frac{d_{f}}{R^{d_{f}}-1} \frac{t_{D}^{\alpha}}{\Gamma(\alpha+1)}-
$$

$$
\begin{gathered}
\pi r_{D}^{\frac{1-\beta}{2}} \sum_{n=1}^{\infty} G_{1}\left(\lambda_{n}\right) t_{D}^{\alpha} E_{\alpha, \alpha+1}\left(-\lambda_{n}^{2} t_{D}^{\alpha}\right) \\
G_{1}\left(\lambda_{n}\right)=\frac{\lambda_{n} J_{v-1}\left(b R^{a} \lambda_{n}\right) J_{v-1}\left(b \lambda_{n}\right) M_{2}\left(\lambda_{n} r_{D}\right)}{J_{v-1}^{2}\left(b \lambda_{n}\right)-J_{v-1}^{2}\left(b R^{a} \lambda_{n}\right)} \\
M_{2}\left(\lambda_{n} r_{D}\right)=J_{v}\left(b r_{D}^{a} \lambda_{n}\right) Y_{v-1}\left(b R^{a} \lambda_{n}\right)- \\
Y_{v}\left(b r_{D}^{a} \lambda_{n}\right) J_{v-1}\left(b R^{a} \lambda_{n}\right)
\end{gathered}
$$

3.4 Exact solution for the flow model of fluid in a finite constant-pressure outer boundary The initial and boundary value problem III that is 
made up of Eqs. (6),(7),(8) and (11) represents the case of constant production rate from a finite constant-pressure outer boundary reservoir. With the application of Laplace transformation to the initial and boundary value III, one obtains the boundary value problem that is made up of the Eqs. (12) (13) and (38)

$$
\left.\bar{p}_{D}\right|_{r_{D}=R}=0
$$

the exact solution of the model III is obtained by using The method similar to the finite closed formation

$$
\begin{aligned}
& p_{D}\left(r_{D}, t_{D}\right)= \\
& \pi \sum_{n=1}^{\infty} G_{2}\left(\lambda_{n}\right) t_{D}^{\alpha} E_{\alpha, \alpha+1}\left(-\lambda_{n}^{2} t_{D}^{\alpha}\right)
\end{aligned}
$$

where

$$
\begin{gathered}
G_{2}\left(\lambda_{n}\right)=\frac{\lambda_{n} J_{v}\left(b R^{a} \lambda_{n}\right) J_{v-1}\left(b \lambda_{n}\right) M_{3}\left(\lambda_{n}\right)}{J_{v-1}^{2}\left(b \lambda_{n}\right)-J_{v}^{2}\left(b R^{a} \lambda_{n}\right)} \\
M_{3}\left(\lambda_{n}\right)=\left[J_{v}\left(b r_{D}^{a} \lambda_{n}\right) Y_{v}\left(b R^{a} \lambda_{n}\right)-\right. \\
\left.Y_{v}\left(b r_{D}^{a} \lambda_{n}\right) J_{v}\left(b R^{a} \lambda_{n}\right)\right] \lambda_{n}(n=1,2, \ldots)
\end{gathered}
$$

is the $n$-th positive root of the following equation

$$
J_{v-1}(b x) Y_{v}\left(b R^{a} x\right)-Y_{v-1}(b x) J_{v}\left(b R^{a} x\right)=0
$$

\section{REFERENCES}

[1] CHANG J., YORTSOS Y. C. Pressure transient analysis of fractal reservoir[J]. SPE Formation Evaluation,1990, 5(1): 31-38.

[2] ACHNA J. A., ERSHAGH I., YORTSOS Y. C. Fractal analysis of pressure transient in the Geysers geothermal field[A]. 17th Annual Workshop Geothermal Reservoir Engineering[C]. Stanford, CA,1992.

[3] ACUNA J. A., ERSHAGH I.,YORTSOS Y. C. Practical application of fractal pressure transient analysis in naturally fractured reservoirs[J]. SPE Formation Evaluation, 1995 10(3): 173-179.

[4] TONG Deng-ke, GE Jia-li. An exact solution for unsteady seepage flow through fractal reservoir[J]. Acta
Mechanica Sinica,1998，30(5): 621-627.(in Chinese)

[5] TONG Deng-ke, CHEN Qin-lei. An exact solution and pressure characteristics of the flow of fluid through fractal reservoir[J]. Journal of Hydrodynamics, Ser.A, 1999,14(2): 201-209. (in Chinese)

[6] TONG Deng-ke, WANG Rui-he. Exact solution and dynamical characteristics of non-Newtonian power-law fluid flow in fractal reservoir.[J]. Journal of Hydrodynamics, Ser.A, 2000,15(3):342-350. (in Chinese)

[7] TONG Deng-ke, WANG Rui-he. The line-source solution and flow analysis of fluid in fractal reservoir[J]. Journal of Hydrodynamics,Ser.B, 2002,14(4):59-65.

[8] FRIEDRICH C. H. R. Relaxation and retardation functions of the Maxwell model with fractional derivatives[J]. Rheol. Acta, 1991,30(2):151-158.

[9] HUANG Jun-qi, LIU Ci-qun. Analysis of general second-order fluid flow in double cylinder rheometer[J]. Science in China, Ser. A,1996,26(10):912-920. (in Chinese)

[10] TAN Wen-chang, XIAN feng, WEI Lan. The exact solution for unsteady Couette flow of generalized second order fluid[J]. Chinese Science Bulletin, 2002,47(16): 1226-1228. (in Chinese)

[11] XU Ming-yu, TAN Wen-chang. Theoretical analysis of the velocity field stress field and vortex sheet of generalized second order fluid with fractional anomalous diffusion[J]. Science in China, Ser. A, 2001,31(7):626-638. (in Chinese)

[12] XU Ming-yu, TAN Wen-chang. The expression of generalized fractional element networks and generalized solution for the constitutive equation of viscoelastic materials $[\mathrm{J}]$. Science in China, Ser. A, 2002,32(8):673-681.(in Chinese)

[13] TONG Deng-ke, SHI Lina. The generalized flow analysis of non-Newtonian visco-elastic fluid flows in porous media[J].Journal of Hydrodynamics,Ser. A, 2005,19(6):695-701. (in Chinese)

[14] PARK H. W., CHOE J., KANG J. M. Pressure behavior of transport in fractal porous media using a fractional calculus approach[J]. Energy Sources,2000, 22(10):881-890.

[15] SONG D. Y.,JIANG T. Q. Study on the constitutive equation with fractional derivative for the vicoelastic fluids-Modified Jeffreys model and its application[J]. Rheologica Acta, 1998, 27:512-517.

[16] GE Jia-li,TONG deng-ke. The mechanics of nonlinear fluid for complex seepage system[M]. Dongying: Petroleum University Press, 1998. (in Chinese)

[17] PODLUBNY I. Fractional differential equations[M]. San Diego: Academic Press,1999.

[18] TONG Deng-ke, CHEN Qin-lei. Generalized flow analysis of non-Newtonian visco-elastic fluid flow through fractal reservoir[J]. Applied Mathematics and Mechanics (English Edition), 1999, 20(12): 1267-1274. 